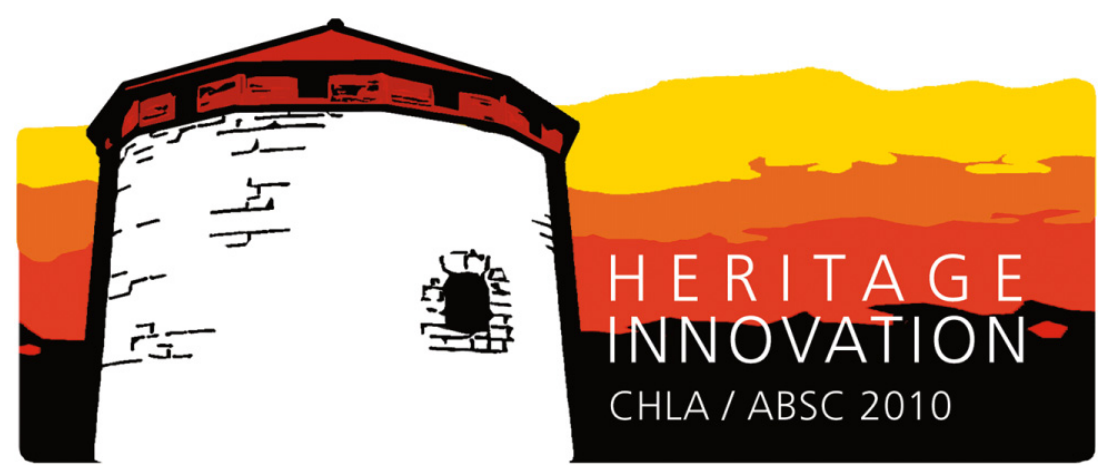

Canadian Health Libraries Association 2010 Conference

7-11 June 2010

Kingston, Ontario, Canada
Association des bibliothèques de la santé du Canada Congrès 2010

\author{
7 au 11 juin 2010 \\ Kingston (Ontario), Canada
}

\title{
Poster abstracts / Résumés d'affiches
}





\title{
SOGC (Society of Obstetricians and Gynaecologists of Canada) and the Museum of Health Care at Kingston - a collaborative approach to the preservation of health care history
}

\author{
Marian Lapham \\ Society of Obstetricians and Gynaecologists of Canada (SOGC), Ottawa, ON, Canada
}

Background: In 2007, the SOGC and the Museum of Health Care at Kingston began a partnership aimed at preserving artefacts representative of the development of obstetrical and gynaecological innovations by Canadians. In undertaking this partnership, both organizations are responding to a critical lack of appropriate repositories for the preservation of the health care sector's material legacy. This joint initiative also represents an opportunity to promote and celebrate health care history in Canada. Program objective: We aim to preserve the history of the ob/gyn specialty, contribute to the ongoing support of the Museum, and promote heritage programming in the health care sector. This program will also encourage specialty societies to recognize themselves as stakeholders in heritage institutions, thereby fostering greater involvement and improved results. Partnership activities to date include the donation of SOGC's instruments to the Museum, a promotional campaign inviting member contributions, and an installation by the Museum at SOGC's National Office showcasing the development of Dr. Jacques-Émile Rioux's bipolar tubal forceps. Future plans include the development of outreach tools, such as travelling displays for annual and regional meetings. Expected outcome: Among the anticipated benefits of the program is a representative collection of obstetrical and gynaecological development that is appropriately housed, preserved and publicly accessible. By encouraging member involvement, we expect to increase publicity and support for the Museum's activity and growth. We also anticipate that success in this partnership will benefit the cultural community by encouraging collaboration between the Museum and other specialty societies.

\section{Honouring our Heritage: The Manitoba Association of Health Information Providers (MAHIP) Chapter History Project}

\author{
Christine Shaw-Daigle, ${ }^{1}$ Lisa Demczuk, ${ }^{2}$ Melissa Raynard, ${ }^{3}$ and Angela Osterreicher ${ }^{4}$ \\ ${ }^{1}$ Carolyn Sifton Helene Fuld Library, University of Manitoba Health Sciences Libraries, \\ Winnipeg, MB, Canada; ${ }^{2}$ Victoria General Hospital, University of Manitoba Health Sciences \\ Libraries, Winnipeg, MB, Canada; ${ }^{3}$ Concordia Hospital, University of Manitoba Health \\ Sciences Libraries, Winnipeg, MB, Canada; ${ }^{4}$ J.W. Crane Memorial Library, Deer Lodge \\ Centre, University of Manitoba Health Sciences Libraries, Winnipeg, MB, Canada
}

Background: The Manitoba Association of Health Information Providers (MAHIP), formerly Manitoba Health Libraries Association, has been in existence since
1976. The membership has been inspired to undertake a history project to create an accessible and dynamic repository of association documents and memories, 
reflecting almost 35 years of chapter activity. Objective: The history project was undertaken with the goal of establishing an open-access digital repository of association documents and supplementary materials and establishing a timeline of association achievement. Methods: A working group from the association was formed to gather, sort and organize documents related to the creation, business and activities of the association. Criteria were developed for the retention of documents. Selected core documents reflecting the course of the association's history were digitized and posted on a wiki developed as the online platform for the repository. Personal reflections about the association were captured and added to the repository. Results: From the initial collection, sorting and organizing of association documents, the MAHIP history project has resulted in the following outcomes: a retention policy for association materials, an organized collection of documents for deposit to the Manitoba Archives and a dynamic, online repository of digitized materials and personal interviews. A timeline of achievement reflecting the highlights of association history has been developed. Discussion: This history project has resulted in an accessible and rich resource for the history of health libraries and health information provision in the province of Manitoba. The project contributes to the heritage of health librarianship in Canada.

\title{
Province-wide catalogue sharing: a developing project
}

\author{
M'hamed Belkhodja \\ Health Science Library, Dr-Georges-L-Dumont Regional Hospital, Moncton, NB, Canada
}

Background: In 2007, the Centre de formation médicale du Nouveau-Brunswick. at the Université de Moncton began a medical teaching program affiliated with Université de Sherbrooke. Soon after, in 2008 the province of New Brunswick merged 8 regional health authorities into 2 health authorities. The division was made largely along linguistic lines. Methods: It was identified that a large portion of the hospital library resources in francophone NB were not readably searchable. This became an issue with influx of students moving around the province and new doctors seeking online resources, particularly in rural areas. A pilot project began with the largest French medical library in the province (Hôpital régional Dr Georges-LDumont) with the integration of their book collection into the Université de Moncton Éloize library catalogue. A costsharing partnership was developed. The holding list was expanded and new listings were added as need be. This publicly available catalogue is now searchable by students and staff from all hospitals and the university community. The second phase is ongoing and involves the integration of holdings from the 3 other medical libraries in the health region: Bathurst, Campbellton and Edmundston. Expected outcome: The end goal will be to set up an automated system for province-wide lending. The poster will detail the steps taken to create a regional health catalogue, the issues encountered and the benefits of this new database. In addition, future plans will be outlined with recommendations welcomed from other CHLA participants.

\section{WorldCat Local in a health sciences environment: one library's experience}

\author{
Shannon Gordon \\ Health Sciences Library, Memorial University, St. John's, NL, Canada
}

Introduction: In 2009, Memorial University completed a reclamation project to fully integrate library holdings into WorldCat. A major motivation behind this was to embark on a pilot project with WorldCat Local, an available discovery layer. Following the reclamation, an eight member WorldCat Local Implementation Committee was created, encompassing systems, cataloguing, public service, electronic resources, serials and circulation expert- ise. In January 2010, this group will begin to assess and evaluate this tool. Memorial's experience speaks to the conference theme of "Honouring Heritage, Inspiring Innovation": can library holdings at Memorial University be successfully discovered via this exciting new tool while honouring relevant and traditional discovery values? Areas to be discussed include: overseeing customization and testing of the WorldCat Local interface; what WorldCat 
Local means for access to health sciences information; and Memorial University Libraries' next steps with this exciting pilot project. Methods: At the time of submission, no formal methodology has been used. Results: Results detailing the functionality of WorldCat Local at Memorial University have yet to be determined. Discussion: Atten- dees will obtain recent and relevant insight and practical advice. This session will be of interest to anyone intrigued by discovery layers and their implications. This session will also offer the perfect opportunity to discuss the various discovery layers being used, or considered, across Canada.

\title{
Combining separate product searches into one to improve efficiency - the experience of the information specialists at the BMJ Evidence Centre
}

\author{
Olwen Beaven \\ BMJ Evidence Centre, London, UK
}

Introduction: Information Specialists at the BMJ Evidence Centre were challenged with an increasing workload of regular updating searches, but with no significant increase in resources to facilitate this. In order to minimise the amount of duplication between related searches for different products, we devised a new search process to integrate and streamline search strategies and appraisal, whilst still delivering different search requirements. Methods: We mapped the different medical conditions covered by the various products and decided which overlapped sufficiently to be grouped together under one all encompassing search. We devised new general search strategies to broaden out all the conditions covered and to accommodate these new groupings. We then instigated a two-tier appraisal approach, first identifying systematic reviews,
RCTs and guidelines on the appropriate condition, before those selected, were appraised again for specific products as required. Results: We have part completed the process of transition to this new search system. Whilst it has been tricky to get things set up and to adjust to this different format, we have found that combining searches together does help streamline processing tasks. Once an update is done for one product, it reduces the work required to update another on the same condition. It also creates a general collection of good quality articles that could be used for further product updates or expansion of condition coverage. Discussion: We are finding this an interesting way to manage increasing search requests and it has expanded the range of searches that the same team can cover.

\section{Nursing questions - searching CINAHL, Medline or both?}

\author{
Debbie Ayotte, Alexandra (Sascha) Davis, and Risa Shorr \\ Ottawa Hospital Library Services, Ottawa, ON, Canada
}

Introduction: Presently there is a large overlap of journal titles in the databases Medline and CINAHL. In addition the ease of use of interface for each database is quite different. Recognizing this overlap and difference of interface, one questions the need to search both databases for all clinical nursing questions. Purpose: To compare results of nursing-related searches conducted in the databases Ovid Medline and CINAHL to determine whether, given time constraints, it is always beneficial to utilize both databases to obtain the highest quality and most relevant search results. Method: Twenty nursing questions will be selected from our literature search database and a search run on each question in Ovid Medline and CINAHL databases using subject headings and keywords. The time required to complete the search in each database will be recorded. The results found will be entered into a Reference Manager database and reviewed for duplication and unique references. Any differences in results will be analyzed for relevancy and quality. Results: To be determined. Discussion: As time is a factor when doing clinical nursing questions in a hospital environment, libraries must regularly evaluate which databases provide the most relevant and quality results in an easily retrievable manner. 


\title{
The missing 2\%: PubMed NOT Medline
}

\author{
David Kaunelis, Kelly Farrah, and Melissa Severn \\ Canadian Agency for Drugs and Technologies in Health (CADTH), Ottawa, ON, Canada
}

Objective: All but approximately $2 \%$ of PubMed citations are found in Medline. Our objective was to establish a search filter for retrieving PubMed citations that are not found in OVID's version of Medline. Methods: Representatives from OVID and the National Library of Medicine (NLM) were contacted to provide background information on the content found in Medline and PubMed. Further information was gathered from web-based sources. Proposed filters were tested by a team of information specialists by comparing the results of identical searches in the two databases. Results: Two search filters were developed. The first was based on PubMed/Medline status fields while an additional filter was based on the PubMed entry date. A search on the term "publisher" in the PubMed status field was found to be effective in identifying the vast majority of PubMed citations not found in Medline. An additional search filter limiting to very recently added PubMed citations was also found to be useful. Discussion: In reality, there is no foolproof way to limit PubMed searches solely to citations that are not found in Medline. There is a considerable flow of information between the two databases before a citation is completely processed. As a result, the status field for an individual citation is updated regularly, making precise searching difficult. However, the above filters were found to work well in identifying unique PubMed citations.

\section{From triage to the big picture: designing a comprehensive information literacy program for a medical faculty}

\author{
Dale Storie and Sandy Campbell \\ John W. Scott Health Sciences Library, University of Alberta, Edmonton, AB, Canada
}

Introduction: Information literacy is highly integrated into the undergraduate medical education program offered by the University of Alberta Faculty of Medicine and Dentistry. However, the Faculty is large and diverse, and also includes medical residencies, graduate research programs in clinical and basic medical sciences departments. Many individuals are remotely located. This project involves a needs assessment to identify gaps in current instruction, a realignment of teaching priorities and the use of asynchronous delivery mechanisms. Methods: Methods included surveying constituents within the Faculty, meeting with key stakeholders and collaborators, analyzing relevant program documents, and conducting a literature review of available research. Results: Full results are forthcoming. Early findings indicate need for an increased focus on alternate modes of delivery, especially online delivery, as well as point-of-need instruction. Clinical and research needs of various groups are now more clearly defined and understood. Discussion: This research identifies existing gaps in instruction and potential improvements. Liaison librarians will now implement an information literacy strategy that ensures that all groups receive the appropriate level of instruction necessary for their learning, research, and practice. Some anticipated changes include strategic delivery of instruction and complementing classroom instruction with more in-person and distance outreach initiatives. A fully integrated online presence is also needed to better accommodate off-campus users and provide assistance at point-of-need. This strategy will be delivered and assessed in staged implementation over the next few years. 


\title{
Finding faculty: the value of partnering
}

\author{
Jessica McEwan ${ }^{1}$ and Lee-Anne Ufholz ${ }^{2}$ \\ ${ }^{1}$ Berkman Library, University of Ottawa Heart Institute, Ottawa, ON, Canada; ${ }^{2}$ Health \\ Sciences Library, University of Ottawa, Ottawa, ON, Canada
}

Introduction: In the autumn of 2008, the Health Sciences Library of the University of Ottawa was invited to participate in the orientation session for the new faculty of the Faculty of Medicine. What has followed is an ongoing partnership in which the library is integrated into the continuing professional development accredited training program offered by the Office of Faculty Development. Methods: Drawing on our experiences with our users, we created a list of possible workshops that would be relevant to members of the faculty in a clinical or research milieu. The Office of Faculty development took our recommendations to their advisory committee who in turn selected three sessions for 2008/09. The Office of Faculty Development packaged, marketed and managed the logistics of the sessions. Each session included a hands- on approach to learning in a computer laboratory with two librarians. It was essential that participants felt their time was spent acquiring and practicing a new skill. Results: Three different sessions were delivered which covered a variety of topics including literature searching, RSS feeds, choosing and using Point of Care Tools, and citation analysis. The partnership promoted our services to an established network of users who responded with enthusiasm. This small group, hands-on format has given us a wealth of usability information and feedback. Discussion: Partnering outside the traditional library affiliations has led to increased visibility. The marketing of these sessions has generated downstream connections to clinical and research based faculty who would otherwise not benefit from our expertise.

\section{Données factuelles en santé mentale: évolution du rôle du bibliothécaire dans la formation des residents}

\author{
Monique Clar \\ Bibliothèque de la Santé, l'Université de Montréal, Montréal, QC, Canada
}

Situation : Dans le cadre du cours « Données factuelles en santé mentale » offert par la Faculté de médecine aux résidents III en psychiatrie, la formation documentaire donnée par la bibliothécaire a évolué au cours des quatre dernières années afin de mieux répondre aux objectifs de ce cours visant à promouvoir une pratique clinique basée sur des données factuelles. Programme : En 2006, les résidents en psychiatrie participent à un atelier de formation de 3 heures sur les ressources documentaires en pratique factuelle, tenu à la Bibliothèque de la santé. En 2007, le professeur responsable du cours « Données factuelles en santé mentale " demande l'ajout d'un atelier pratique avancé de 3 heures afin que les résidents explorent davantage les ressources utilisées lors de l'atelier de formation. Depuis 2008, la bibliothécaire participe aussi activement à la séance où les résidents présentent orale- ment en classe les résultats de leur recherche documentaire sur une question clinique de leur choix. Résultats : La bibliothécaire participe maintenant à 9 heures sur un total de 45 heures de cours. Cette collaboration présente des avantages pour tous. La participation de la bibliothécaire aux présentations orales des résidents lui a permis de mieux comprendre la façon dont les résidents abordent une question clinique et utilisent les outils de recherche documentaires. Les discussions entre les résidents, le professeur et la bibliothécaire ont également permis de mieux cerner le rôle et l'apport du bibliothécaire au sein d'une équipe multidisciplinaire en santé mentale. Conclusion : La participation de la bibliothécaire à ce cours est maintenant bien établie. Il serait intéressant de pouvoir éventuellement offrir une formation semblable aux résidents des autres spécialités médicales. 


\title{
Inspiring innovation through collaboration: a library workshop for biomedical engineering graduate students
}

\author{
Michelle Baratta ${ }^{1}$ and Maria Buda ${ }^{2}$ \\ ${ }^{1}$ Engineering and Computer Science Library, University of Toronto Libraries, Toronto, ON, \\ Canada; ${ }^{2}$ Dentistry Library, University of Toronto Libraries, Toronto, ON, Canada
}

Introduction: Inspiring Innovation through collaboration Instruction in an interdisciplinary setting has become an exciting challenge in academic health science libraries. The authors would like to share their experience in combining subject expertise of Engineering and Dentistry to create an instructional workshop for a Biomedical Engineering graduate course. Background: The first year graduate course BME 1450 (Bioengineering) is mandatory in the Biomedical Engineering Program, which leads to a Masters of Applied Science (M.A.Sc.). Admission to the M.A.Sc. requires a bachelor in engineering, medicine, dentistry, or other physical or biological sciences. The educational background of the students in this program varies and it is therefore important for them to become familiar with the available resources in both medicine and engineering. The course itself, BME 1450, requires the students to form groups, conduct research on a bioengineering topic and present the results in a poster. Methods: The librarians instructed a one and a half hour workshop to cover searching medical (PubMed, Ovid Medline, Cochrane Library) and engineering databases (IEEE, Compendex, Scopus and Web of Science). Discussion: Exposure to resources in both fields provided a broader knowledge of available tools for their research needs. It was also a great learning experience for the novice librarians to step out of the comfort zone and apply their subject expertise in an interdisciplinary setting.

\section{A consumer health collection in an academic library: transformation to a student-centred collection}

\author{
Jill Boruff \\ Life Sciences Library, McGill University, Montreal, QC, Canada
}

Introduction: The Wendy Patrick Health Information Collection at McGill University is named and funded in honour of a deceased nursing librarian. Started in the 1990s, the collection has its roots in a public health clinic and is now housed as a separate collection in the Life Sciences Library at McGill. Its original focus on resources for the public of Montreal has been hindered by the library policy that does not allow for books to circulate to the public without a paid membership. This poster examines how to best honour the collection established in Wendy Patrick's memory while promoting the innovative use of the current collection. Methods: The librarian undertook a project in 2009 to update the collection through the deselection of 200 out-of- date books and the purchase of new 70 titles. These new materials, and most new titles since, were chosen to focus on the student population and their own health concerns. Results: Through the librarians' observations, these new materials seem to be popular, though meaningful circulation statistics are not yet available. Discussion: Many questions remain about this collection. Would integrating it into the general collection increase its use or make it more difficult to find materials? Should the collection be given a more descriptive name? How can the collection be best advertised to the McGill students and staff? This poster is meant to encourage a dialogue with other health librarians on the role of a consumer health collection in an academic library. 


\title{
Richmond Health Literacy Project: better health through better understanding of health information
}

\author{
Belinda Boyd, ${ }^{1}$ Dace Starr, ${ }^{2}$ and Shannon Long ${ }^{3}$ \\ ${ }^{1}$ Vancouver Coastal Health, Richmond, BC, Canada; ${ }^{2}$ Richmond Public Library, Richmond, \\ BC, Canada; ${ }^{3}$ Vancouver Coastal Health Authority, Richmond Hospital, Richmond, BC, \\ Canada
}

Introduction: A September 2007 Health Literacy Report from the Canadian Council on Learning indicated Richmond has one of the lowest health literacy rates in the country. In response, the Richmond Health Advisory Committee, residents who advise senior management of Vancouver Coastal Health (VCH) on ways to consult with the community, hosted a public forum on health literacy in 2008. A partnership between VCH and the Richmond Public Library (RPL) was established to identify ways that health care workers could promote health information that could support patients to manage their chronic conditions. This project had two goals; (1) gather recommended titles from physicians and health care clinicians to build public and hospital based library collections of materials that will support patients in the management of their chronic conditions, (2) help patients develop skills in accessing, understanding and using health information to better manage their chronic conditions by asking health practitioners to refer patients to use the libraries resources. Methods: Physicians and health practitioners were targeted through meetings and other methods and provided with: bookmarks; flyers; prescription pads; and a dedicated health webpage on RPL site that allows practitioners to recommend books online at any time. Conclusion: Formally launched in April 2009 the RPL and the Patient/Family Library at Richmond Hospital added space and increased access to health related materials and online access to reputable health sites. To date both libraries have built an impressive collection of resources to support residents, patients and family members in the management of chronic conditions.

\section{Using RSS feeds to create a targeted current awareness website}

\author{
Shannon Long \\ Vancouver Coastal Health Authority, Richmond Hospital, Richmond, BC, Canada
}

Introduction: The Vancouver Coastal Health (VCH) library team, which serves a large geographic area in southwestern British Columbia, wanted to reach out to the allied health professionals in their health authority who were noted to be infrequent library users. Description: The library team created a special website comprised almost entirely of content from RSS feeds. The site contains current health headlines from respected news sources and provides a single place to view the most recent articles and guidelines related to each allied health discipline. The leaders of each professional group determined the topics they needed to be kept up to date on then the library staff performed focused literature searches using MEDLINE, CINAHL and PsycINFO. The searches were saved as alerts and exported using RSS Feeds which were then loaded onto the website. The users see only the five most recently indexed articles on the topics they determined were most relevant to their discipline and their work within $\mathrm{VCH}$. The content of the site is automatically populated by search alerts and daily news feeds so it is always current and very easy to maintain. Outcomes and discussion: What started out as a small idea has quickly grown into a great new service and a special website that is truly relevant and patron driven. To date, the site contains over 90 search alerts for 15 different professional groups. The library staff continue to receive requests for additional topics and are looking forward to expanding the service to other health disciplines (http://www.vchlibraryservices.blogspot.com). 


\title{
Top 10 Canadian consumer health websites - updated 2010
}

\author{
Tom Flemming, Mary Anne Howse, ${ }^{1}$ Erica Lee, ${ }^{2}$ Lily Mac,, ${ }^{2}$ Marg Muir, ${ }^{3}$ and \\ Elizabeth Puckering 4 \\ ${ }^{1}$ Library Services, Women's College Hospital, Toronto, ON, Canada; ${ }^{2}$ AIDS Committee of \\ Toronto, Toronto, ON, Canada; ${ }^{3}$ Sunnybrook Health Sciences Centre and Trillium Health \\ Centre, Toronto, ON, Canada; ${ }^{4}$ Mississauga Library System, Mississauga, ON, Canada
}

Introduction: In a 2004 survey of 3,000 Canadians about their Internet use, the Canadian Internet Project found that $54.4 \%$ of respondents searched for health information. By 2007 the number had jumped to $70 \%$. Both surveys found that most people use a general search engine such as Google or Yahoo. Health information providers know that general search engines are not the most effective way to locate quality health information websites. Librarians and other health information providers know about specific health portals, and about how to assess the quality of information in a website. Methods: In 2006, a subcommittee of the Consumer Health Information Provider's Interest Group (CHIPIG) surveyed health science librarians, public librarians, and health promotion specialists for recommendations of quality Canadian consumer health websites. A list of 10 websites was developed and presented in a round-table discussion at the CHLA Conference in Vancouver. Three of the websites on that list no longer exist, including the Canadian Health Network. CHIPIG repeated the survey in 2010 and has created an updated list. Once again, we asked librarians and other health information providers to submit their favourite Canadian consumer health information websites for consideration. The criteria we used include credibility, content, audience, currency, and disclaimers. Results: We will distribute the results to librarians and health promotion specialists so that they can recommend the websites to consumers and to health care providers.

\section{Reaching in and out: bridging the gap in serving multi-lingual communities}

\author{
Rita Kang, Po-Lin Cheung-Leung, Valeria Raivich, and Miu Lin Wong \\ Toronto Western Hospital, Toronto, ON, Canada
}

Introduction: The health care environment requires hospital libraries to develop strategies for providing consumer health information (CHI). The assumption is that consumers who are well informed will become an empowered partner in their health and the system. This is a particular challenge for people who have limited English proficiency (LEP). Language barriers have adverse effects on: access to health care, quality of care, patient safety, patient outcomes, patient rights, and provider satisfaction (Divi, Koss, Schmaltz \& Loeb, 2007). The objective of this poster is to share successful strategies in removing language barriers to receiving consumer health information. Methods: Tools, projects and services provided by a patient and family library in a hospital/multicultural community setting in Toronto, Ontario Canada will be described and illustrated. The Toronto Western Hospital patient and family library provides services to patients, families, visitors and the community. Consumer Health information is provided by a Librarian, Health information Specialist and Community Health Educator. We provide over 420000 services per year. Feedback and evaluation is central to our services. Evaluative strategies and results of this data will be highlighted. Discussion: The overall strategies outlined in the poster are aimed to encourage organizations to plan and develop programs with the intent to address health literacy and provide multi-lingual access to consumer health information. The implications for policy, delivery or practice include: effective collaboration, community outreach/partnership, events/ Initiatives that promote health literacy and education, multi-lingual collections, use of technology, utilization of multi-lingual staff, and evaluation and feedback. 


\title{
Library advocacy at the bedside and the boardroom
}

\author{
Renee Reaume, ${ }^{1}$ Elizabeth Aitken, ${ }^{1}$ and Susan Powelson ${ }^{2}$ \\ ${ }^{1}$ Health Information Network, University of Calgary, Calgary, AB, Canada; ${ }^{2}$ Health Sciences \\ Library, University of Calgary, Calgary, AB, Canada
}

Advocacy efforts are described within the Health Information Network: a strategic partnership between the University of Calgary and the former Calgary Health Region and the former Alberta Cancer Board. Creating awareness and understanding of the Health Information
Network led to significant opportunities for innovation and participation on research projects including a six month clinical librarian trial. Also, through the partnership structure advocates are cultivated throughout the organization.

\section{Measuring the impact of library service in a knowledge exchange network}

\author{
Jan Figurski ${ }^{1}$ and James Conklin ${ }^{2}$ \\ ${ }^{1}$ SHRTN Library Service, Baycrest, Toronto, ON, Canada; ${ }^{2}$ Applied Human Sciences, \\ Concordia University, Montreal, QC, Canada
}

Introduction: The Seniors Health Research Transfer Network (SHRTN) is a knowledge exchange network that seeks to improve the health and health care of seniors in Ontario. All parts of the network are continually challenged to identify ways to demonstrate value and measure impact. Methods: The Library Service developed a survey for measuring client satisfaction, perceived value, and the impact that the library products and services had on the practice and decision-making of clients. This survey was distributed to a large, randomly selected cross-section of clients. Those respondents that indicated that the Library Service had had an impact on their practice and decision-making were contacted for a semi-structured follow-up interview. The interviews were used to construct narratives expanding upon the impact described in the survey. Results: Clients are overwhelmingly satisfied with the products and services they receive and place a high value on them. The Library Service is having a significant impact on clients practice and decision-making. Discussion: The impacts described can be categorized into a number of useful areas that provide a basis for strategic planning, for understanding the impact of SHRTN as a knowledge exchange network, and provide a basis for ongoing improvement and action. They make it clear that the library service is one of the main pillars of this knowledge exchange network. Conclusion: The survey and structured interview methods used in this evaluation process may be of some use to other library networks seeking new ways to demonstrate value and to measure impact. 


\title{
An innovative method reveals the impact of clinical information retrieved from electronic knowledge resources
}

\author{
Roland Grad, ${ }^{1}$ Vera Granikov, ${ }^{2}$ Pierre Pluye, ${ }^{3}$ Janique Johnson-Lafleur, Shu Zhang, ${ }^{3}$ \\ Gillian Bartlett, ${ }^{3}$ Vinita D'Souza, ${ }^{4}$ and Bernard Marlow ${ }^{5}$ \\ ${ }^{1}$ McGill University, Montreal, QC, Canada; ${ }^{2}$ Information Technology Primary Care Research \\ Group, Herzl Family Practice Centre, Montreal, QC, Canada; ${ }^{3}$ Department of Family \\ Medicine, McGill University, Montreal, QC, Canada; ${ }^{4}$ Information Technology Primary Care \\ Research Group, Department of Family Medicine, McGill University, Montreal, QC, Canada; \\ ${ }^{5}$ College of Family Physicians of Canada, Mississauga, ON, Canada
}

Introduction: We know little about the impact of specific information objects retrieved by health professionals from electronic knowledge resources. Inspired by the 'Acquisition-Cognition-Application' model of information use, we developed the Information Assessment Method (IAM). We examined the use and validity of IAM linked to searches conducted by family physicians in practice. Methods: This is a quantitative component of a mixed methods study. Over one year, 40 practicing family physicians searched Essential Evidence Plus ${ }^{\circledR}$ and rated their information hits using IAM. Acquisition was operationalized as seven reasons for searching. Cognitive impact was a construct comprised of ten items and 8 factors. Application was based on whether the information was or will be used for a specific patient. Results: 1,767 searches resulted in 3,300 rated information hits (average 44 rated searches and 83 rated hits per physician). Acquisition: Most searches (75\%) were done for more than one reason; most frequently, to address a clinical question (74\%). In 1,336 searches $(76 \%)$, the search objective was met. Cognition: Most frequently, ratings of positive cognitive impact were about confirmation ("this information confirmed I did (am doing) the right thing", 46\%) and reassurance ("I am reassured", 45\%). Ratings of negative cognitive impact were rare $(4 \%)$. Application: 1,708 rated hits (52\%) were (or will be) used for a specific patient. IAM guided interviews will reveal how clinical information was used for specific patients. Conclusion: The "Acquisition-Cognition-Application" model is supported. IAM, for systematic assessment of information objects, can reveal the impact of an electronic knowledge resource. (*Outline of the topic: Inspired by the "Acquisition-Cognition-Application" model of information use, an innovative method revealed the impact of clinical information retrieved by Canadian family physicians.)

\section{Roles for hospital librarians during a pandemic: electronic information monitoring and the provision of synthesized updates to incident management personnel}

\author{
Sandra McKeown \\ Health Sciences Library, London Health Sciences Centre, London, ON, Canada
}

Purpose: There is limited information available that describes how librarians have assisted health care administrators in planning and responding to a pandemic. This poster describes the role of hospital librarians during the recent breakout of the $\mathrm{H} 1 \mathrm{~N} 1$ influenza virus to support the integrated pandemic influenza plan for London hospitals.
Setting: Librarians at both London Health Sciences Centre (LHSC) and St. Joseph's Health Care London (SJHC) worked together to provide daily, and then later weekly, email updates that were sent to incident management personnel from April to December, 2009. Method: Current, reliable information was compiled from key 
government and health organization websites as well as online news centers. Email updates reported new and total numbers of confirmed cases and related deaths as well as new guidance documents, health notices, situation updates, and selected news items. Results: The information compiled in the emails was used to update the pandemic management team, senior leadership team, Medical Advisory Committee and the Boards of Directors at LHSC and SJHC. The number of H1N1 cases and deaths reported in the email updates facilitated the tracking of the pandemic viral spread for incident management personnel. The excerpts of news items allowed health care administrators to anticipate media questions as well as predict and alleviate staff anxiety. Discussion: In addition to email updates, librarians conducted literature searches for staff on pandemic related topics as requested. The recent $\mathrm{H} 1 \mathrm{~N} 1$ pandemic provides an opportune time for librarians to reflect on current and potential roles during a pandemic and to engage health care administrators in conversation as appropriate.

\title{
Establishment of an internet-accessible, Scalable Gross Anatomy and Histology Image Catalogue (SGAHIC) for modern medical and health science education
}

\author{
Stephen Pang, ${ }^{1}$ Conrad Reifel, ${ }^{1}$ Les MacKenzie, ${ }^{1}$ Ron Easteal, ${ }^{1}$ R. David Andrew, ${ }^{1}$ \\ Richard Hunt, ${ }^{1}$ and Shakeel Virk ${ }^{2}$ \\ ${ }^{1}$ Department of Anatomy \& Cell Biology, Queen's University, Kingston, ON, Canada; \\ ${ }^{2}$ Department of Pathology and Molecular Medicine, Queen's University, Kingston, ON, \\ Canada
}

Introduction: Anatomy is an essential component of medical and health science education. Over the past 2-3 decades, there has been a shift in the reduction of basic science contents, including Anatomy, in medical and health science educational programs. As well, the method of delivery of these subjects has changed from a traditional lecture-style to combined didactic, self-directed and/or teambased learning modalities. Methods: To better serve our students, a Scalable Gross Anatomy and Histology Image Catalogue (SGAHIC) was established. This collection contains several thousand images of Gross Anatomy,
Neuroanatomy and Histology captured from the specimens held in the Anatomy Learning Centre at Queen's University. Gross Anatomy and Neuroanatomy images can be enlarged to about 5-8 times and Histology images 400 times their original size. Conclusion: This collection of images not only allows our students to gain access to this learning resource on a $24 / 7$ basis as long as there is an internet connection, but also web-based distant Anatomy and Histology courses can be readily developed to serve students from other universities in Canada and abroad. SGAHIC is sponsored by Bracken Health Sciences Library at Queen's University.

\section{Forging new directions in scholarly communications}

\author{
Sam Kalb \\ Queen's University Library, Queen's University, Kingston, ON, Canada
}

Introduction: The complexity of research in the $21 \mathrm{st}$ century, coupled with the transformation of information technology and the heightened scrutiny of responsible conduct of research issues, has presented challenges with effective knowledge management. The development of new avenues for open access to scholarly digital content and the growing demand from public funding bodies for open access to funded research, offer great opportunities and further challenges for researchers and librarians. This poster demonstrates a program of new initiatives by Queen's University Library (QUL) to address these challenges. Methods: (1) identifying and integrating access 
to scholarly open access journals and databases into the suite of resources offered to the Queen's community; (2) developing new services to preserve and disseminate Queen's scholarship in collaboration with our academic partners. Results: QUL now (1) hosts a growing number of open access peer-reviewed journals in the OJS @ Queen's service; (2) offers a digital repository, QSpace, for the dissemination of faculty and student research; (3) exposes
Queen's researchers to a wide range of scholarly open access resources. Discussion: Acceptance of these new directions in scholarly communication has been slowly growing. There is still considerable resistance and opposition within the academic community. Maintaining quality of research and measurement of impact factors in an open access environment are major areas of concern and ongoing discussion.

\title{
Optimizing training of new health librarians to support evidence-based practice and knowledge translation
}

\author{
Jessie McGowan, ${ }^{1}$ Alla E. lansavichene, ${ }^{2}$ Peggy O'Neil, ${ }^{3}$ Tamara Rader, ${ }^{4}$ and \\ Margaret J. Sampson ${ }^{5}$ \\ ${ }^{1}$ Institute of Population Health, University of Ottawa; Faculty of Medicine, University of \\ Ottawa; Ottawa Hospital Research Institute, Ottawa, ON, Canada; ${ }^{2}$ London Health Sciences \\ Centre, London, ON, Canada; ${ }^{3}$ Health Sciences Library \& Student Affairs, London Health \\ Sciences Centre, London, ON, Canada; ${ }^{4}$ Institute of Population Health, University of \\ Ottawa, Ottawa, ON, Canada; ${ }^{5}$ Children's Hospital of Eastern Ontario, Ottawa, ON, Canada
}

Introduction: Health libraries need to be prepared to undertake the challenge of hiring and training new personnel who will be able to competently participate in translating available knowledge into clinical practice and supporting evidence-based medicine in everyday decision-making process. Methods: The challenges that exist as a result of expected retirements in the profession and potential strategies including ensuring a viable workforce through the development of a training program for new health librarians are described and reviewed. Librarians sometimes do not learn all skills that they need in school to work in a health care team. The knowledge base of the health librarians and its application into practice are important parts of the service provision to the teams of health care providers. Some of these key skills are vital, as, for example, an incomplete literature search or omission to consult relevant sources could affect the evidence-based decision making and produce results leading to bias or even medical error. Results: There are opportunities to improve the knowledge base of health librarians to support evidence-based decision making by incorporating the elements of evidence-based practice and knowledge translation concepts into a new curriculum. Discussion and conclusions: Training of the new health librarian is a crucial to ensure that the skills are learned and developed. A curriculum needs to employ a variety of methods and resources, so that the new librarian can deal effectively with the challenges of the work within the particular health care environment in the least amount of time. 